\title{
Inhaltsverzeichniss des sechzigsten Bandes.
}

Nova methodus, aequationes differentiales partiales primi ordinis inter numerum variabilium quemcunque propositas integrandi. Opus posthumum auctore C. G. J. Jacobi. . . . . . . . . . . . . . . . . . Seite 1

Ueber die Darstellung der Curven durch Krummung und Torsion. Von Herrn R. Hoppe. . . . . . . . . . . . . . . . . . . . . . . - 182

Note sur les cubiques gauches. Par M. L. Cremona Bologne. . . . . - 188

Ueber das Pfaffsche Problem. Erste Abhandlung. Von Herrn A. Clebseh zu Carlsruhe. . . . . . . . . . . . . . . . . . . . . . -193

Die Lameschen Functionen verschiedener Ordnungen. Von Herrn E. Heine in Halle. . . . . . . . . . . . . . . . . . . . . . . -252

Sur la transformation du troisiàme ordre des fonctions elliptiques, extrait d'une lettre de M. Hermite a l'éditeur. . . . . . . . . . . . . - 304

Zerlegung der.Bedingung für die Gleichheit der Hauptaxen eines auf einer Oberfläche zweiter Ordnung liegenden Kegelschnittes in die Summe von Quadraten. Von Herrn Otto Hesse zu Heidelberg. . . . . . . . . - 305

Sur les surfaces gauches du troisième degre. Par M. L. Cremona a Bologne. - 313 Ueber die Anziehung einer von zwei ähnlichen Flächen zweiten Grades begrenzten Schale. Von Herrn Mehler zu Fraustadt. . . . . . . . - 321

Ueber eine Eigenschaft der Kugelfunctionen. Von Herrn A. Clebsch zu. Carlsruhe. . . . . . . . . . . . . . . . . . . . . . . -343

Sur une formule relative à la thérie des nombres. Par M. Emile Mathieu a Paris. . . . . . . . . . . . . . . . . . . . . . . . - 351

- Tables des formes quadratiques binaires pour les déterminants négatifs depuis $D=-1$ jusqu'à $D=-100$, pour les déterminants positifs non carrés depuis $D=2$ jusqu'à $D=99$ et pour les treize déterminants négatifs irréguliers qui se trouvent dans le premier millier. Par M. A. Cayley a Londres. 
Note sur l'élimination. Par M. A. Cayley a Londres. . . . . . . . . Seite 373

Ueber die richtige Werthbestimmung der Constante des Integrallogarithmus.

Von Herrn L. Oettinger zu Freiburg i. B. . . . . . . . . . . . - 375

Ueber die Identität der Prismatoide mit den Trapezoidalkörpern. Von Herrn

E. F. August. . . . . . . . . . . . . . . . . . . . . - 377

Zur Lebensgeschichte des Mathematikers Ludwig Immanuel Magnus. . . . - 379

Inhaltsverzeichniss der Bände 51 bis 60 . . . . . . . . . . . . . - 382 